\title{
PIRFORMANCE OF LINEAR PREDICTION ANALYSIS ON SPEECH WITH ADDITIVE NOISE
}

\author{
B. Yegnanarayana and T.K. Raja
}

Department of Blectrical Communication Engineering Indian Institute of Science Bangalore-560012, India

\section{ABSTRACT}

We shall present in this paper the results of investigations on the performance of Iinear Prediction (IP) anelysis of speech when the signal is corrupted with additive white noise. In particular we shall discuss the effect of noise on various parameters derived from IP analyais. Theoretical justification for the observed results is given.

\section{INTRODUCTION}

Noise is the single most important factor that ultimately determines the performance efficiency of as speech signal processing system 1 . Speecil produced under normal envîronment will have different signal to noise ratios (S/N) in different segments depending upon the level of signal in each segment as for example voiced segments will have higher $S / N$ compared to unvoiced segments. Iinear prediction analysis may be used for transmission of speech information even when speech is corrupted with nois $\theta$ because temporal variations of some spectral features are preserved and the high redundancy of language supplies the missing information. But for applications such as automatic speech and speaker recognition and speech aids to handicapped, the features extracted by IP analysis depend critically on the quality of speech signal. We shall discuss in this paper the effect of $S / N$ of analysis segment on parameters obtained through ip analysis.

\section{IINEAR PREDICTION ANALYSIS}

IP analysis is performed on speech segments corrupted with additive noise. The noise signal is obtained by adding the samples of the output of a random noise generator to speech samples. The sampling is done at $10 \mathrm{KHz}$. IP coefficients obtained by solving the auto- correlation normal equations are used to derive the inverse filter response of the vocal tract. Inverse filter responses for a single resonator output and for roiced speech segments are shown in Figs. 1 to 3 . The responses are plotted with $S / N$ as a parameter. Figs. 4 and 5 show variation of IPCs with $S / N$ for the two vowel segments.

\section{DISCUSSION}

It is interesting to observe that the inverse filter response predicts the resonance frequencies reasonably well even for low $S / N$. But the bandwidhs of the resonance peaks are progressively increased as $S / N$ is reduced. The explanation for such a behaviour can be found. in spectral approximation in IP analysis [2]. The model spectirum approximates the envelope of signal spectrum with higher accuracy of approximation at peaks of the envelope. Addition of white noise merely adds noise spectrum to signal spectmum thus reducing the dynamic range of the overall spectmum without affecting the resonance peaks of the signal spectrum. For a given dynamic range the minimum resonance bandwidth is fixed as can be seen from the expression for the frequency response of a single resonator:

$$
|H(j w)|^{2}=\frac{1}{\left(w^{2}-w_{0}^{2}\right)^{2}+4 \alpha^{2} w^{2}}
$$

where $w_{0}$ is the resonance frequency and $\alpha$ is the half power bandwidth. At resonance $w=w_{O}$ ?

$$
\left|H\left(j w_{0}\right)\right|=\frac{1}{4 \dot{\alpha}^{2} w_{0}^{2}}
$$

At large frequencies $w \gg w_{0}$, and

$$
|H(j w)|^{2}-\frac{1}{w^{4}}
$$




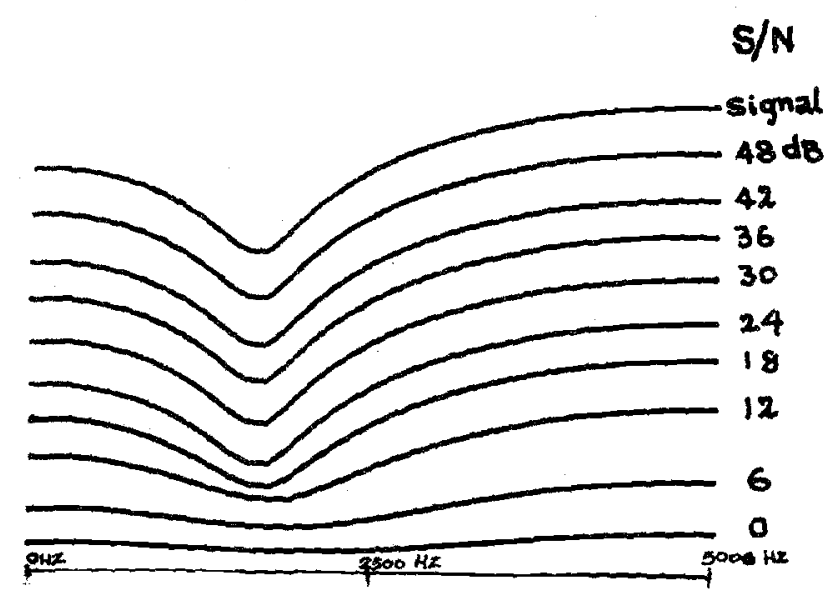

Fig. 1: Inverse filter responses for a single resonator model (Resonance frequency $=1500 \mathrm{~Hz}$ and bandwidth $=150 \mathrm{~Hz}$ )

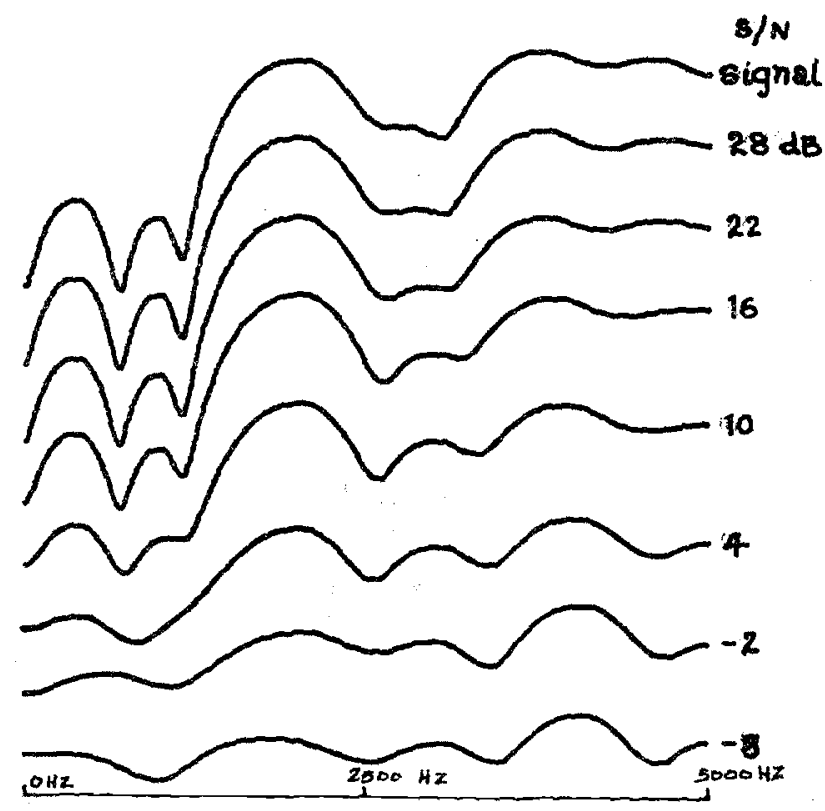

Fig. 2: Inverse filter responses for the vowel segment $|a|$.

Let $w_{1}$ be the highest frequency of interest. IThen the dynamic range a is given by

$$
d=\left(\frac{1}{4 \alpha^{2} w_{0}^{2}}\right) /\left(\frac{1}{w_{1}^{4}}\right)=\frac{w_{1}^{4}}{4 \alpha^{2} w_{0}^{2}}
$$

Since wo is estimated correctly by ITP analysio and $w_{1}$ is fixed, the dymamic range is inversely proportional to square of bandwidth. Therefore if $d$ is rediced bandwidth is automatically increased as can be seen in Fig. 1 .

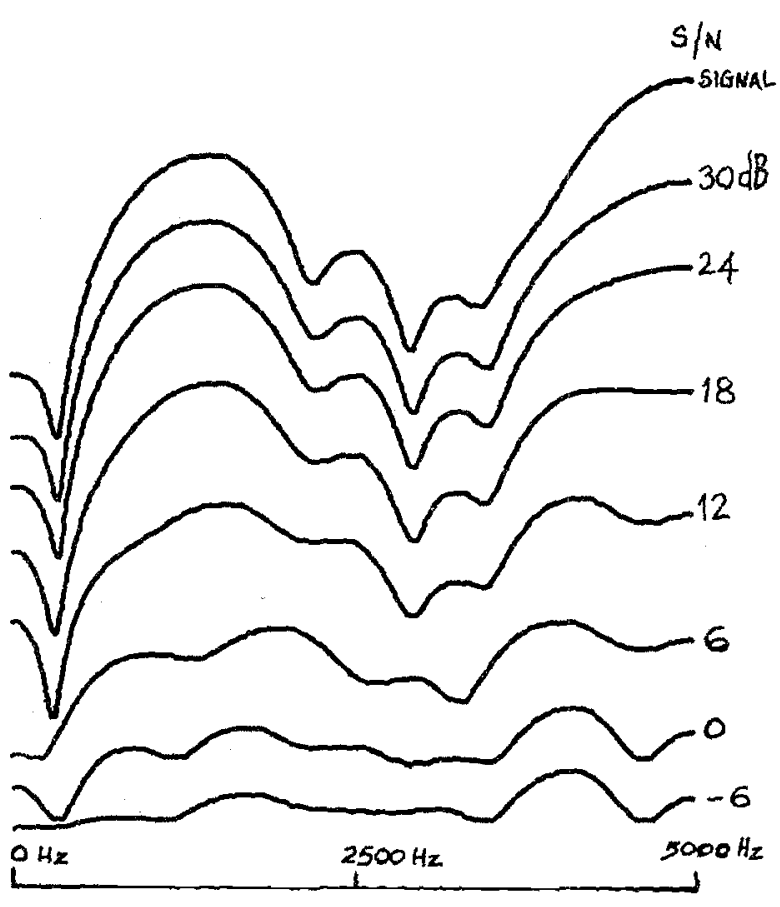

Fig. 3: Inverse filter resporse for the vorrel segment $|i|$.

For speech signal higher formants are not generalily affected as much as the first formant when $S / N$ is reduced. whis is because the first formant has much lower bandwidth and it requires large dynamic range for accurate estimation. In fact better estimates of higher formants are obtained by reducing the dynamic range which is usually realized by providing high frequency emphasis at preprocessing stage. In Fig.2 higher formants are clearly resolved when $s / N$ is lower to about $10 \mathrm{~dB}$.

Al though formant frequencies are estimated correctly even under poor $S / N$ conditions it is difficult to identify them from LP magnitude spectrum by peak picking methods especially when the peaks are close to each other or when the bandwidths are large. Derivative of IP phase spectrum can be effectively usea for this purpose $[3]$ as shown in Fig. 6 for a two resonator model.

The above discussion indicates that IP vocoders should perform satisfactorily even for noisy speech as the temporal variations of formant frequencies are preserved. However when LPCs or related parameters such as reflection coefficients and area functions are used in speech recognition or speaker verification systems, additive noise increases the 


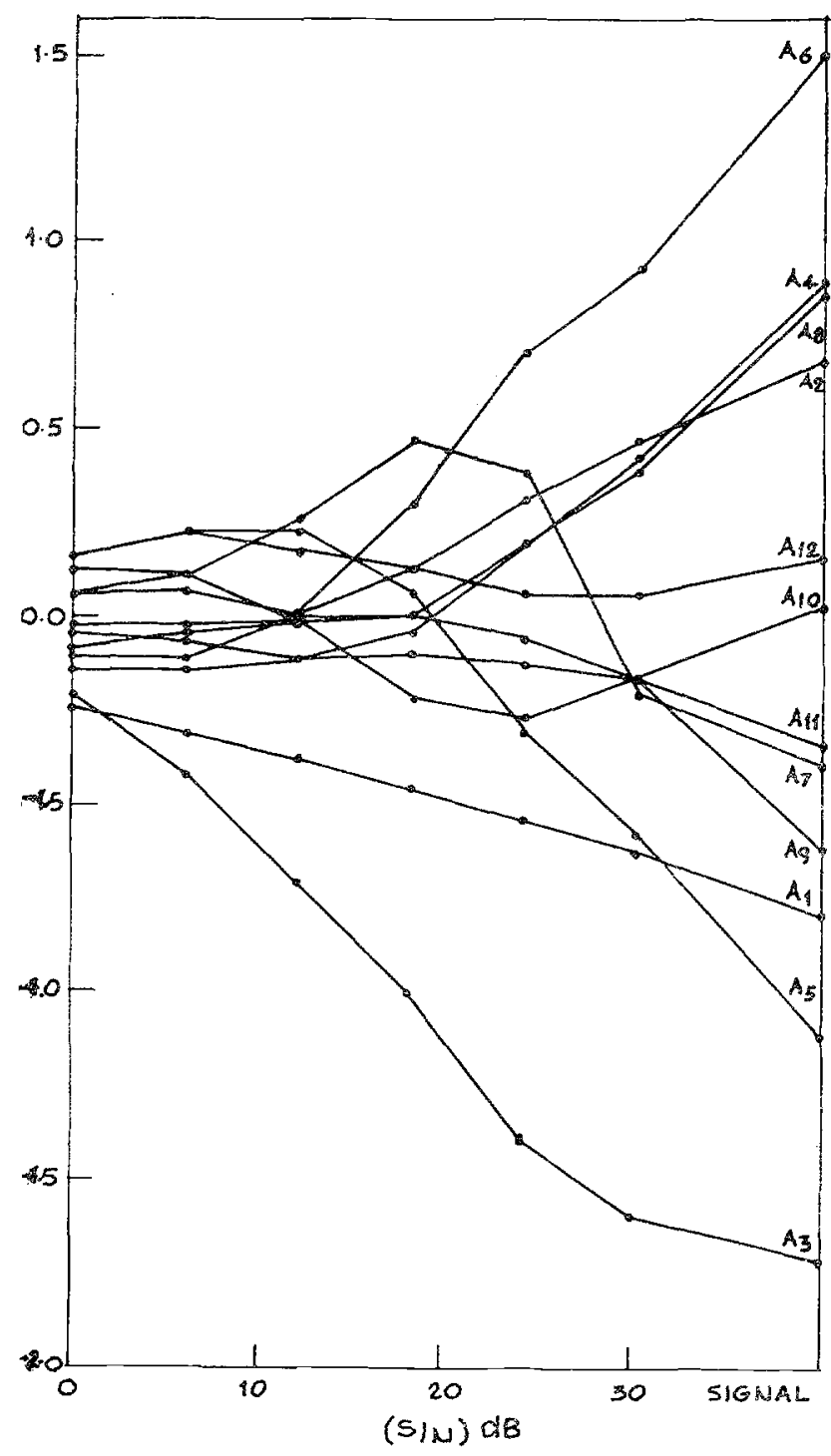

Fig. 4: IPCs for the rowel segment|i|

intra-variation significantly making it difficult to design classifiers for pattern recognition. Typical variations

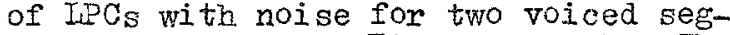
ments are shown in Figs. 4 and 5 . The changes in LPCs even at $30 \mathrm{~dB} S / N$ is significant and the ordering the coeffi. cients is disturbed as noise level increases. One of the important applications of IP analysis is to obtain nonuniform acoustic tube model for rocal tract in terms of area functions. When such models are used as aids to handicapped the noise level becomes a critical factor.

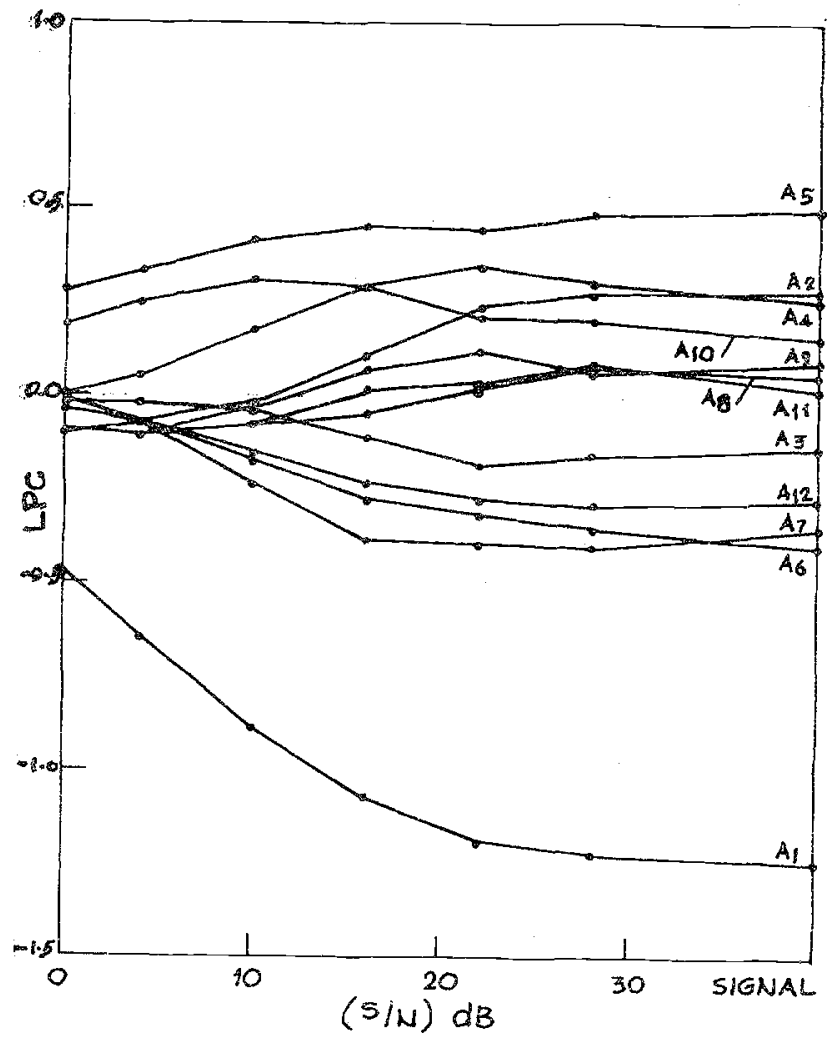

Fig. 5: IPGs for the vowel segment $|a|$.

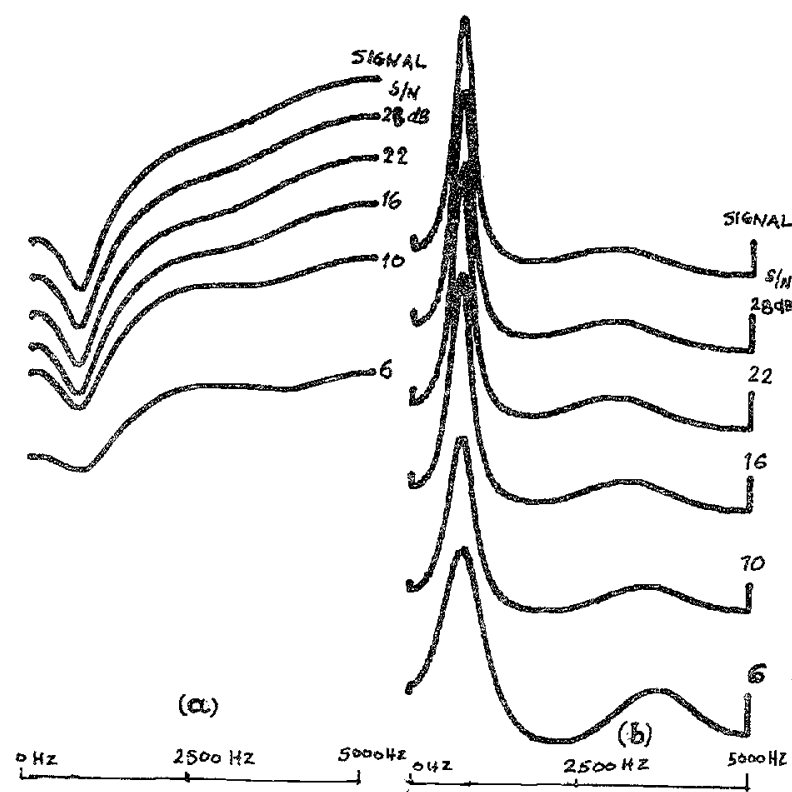

Fig.6: Formant extraction using IJP phase spectrum

(a) Frequency response

(b) Differentiated phase spectrum 


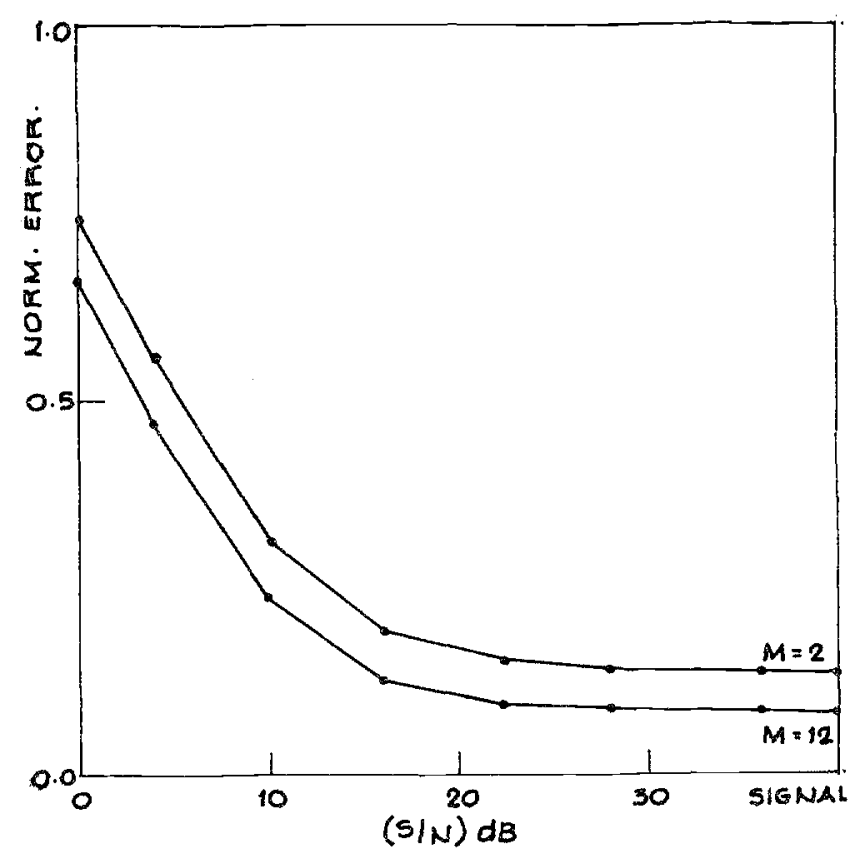

Fig.7: Normalized error for the vowel segment $|a|$

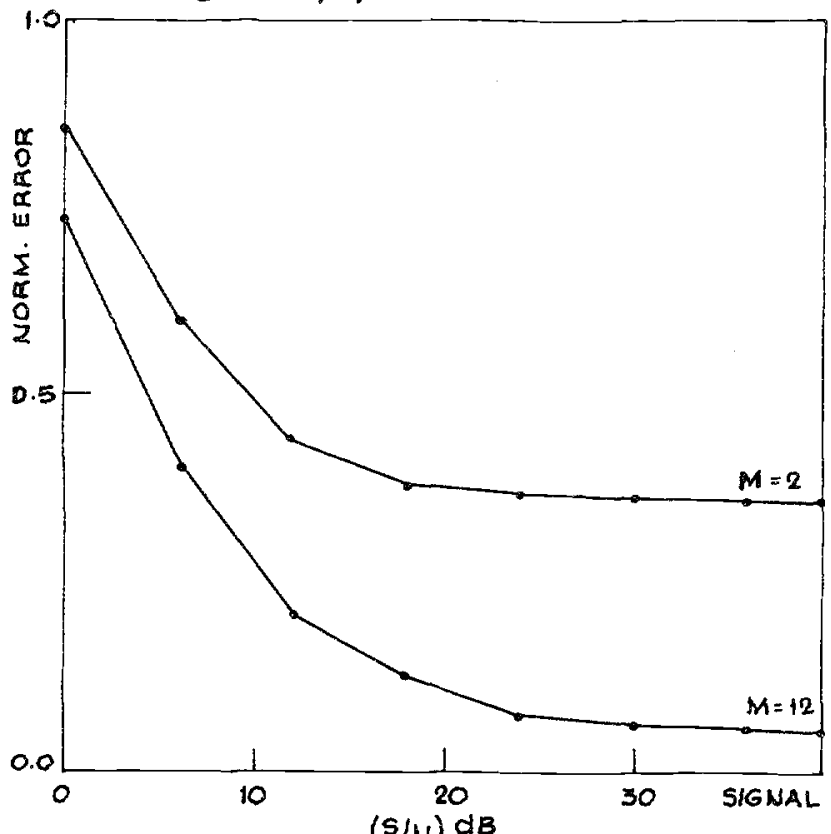

Fig. 8: Normalized error for the vowel segment $|i|$

Another useful parameter is the normalized error which is plotted for different $S / N$ in Figs. 7 and 8 for two vowel segments. Although the general behaviour depicted is to be expected, it is interesting to note that the error increases steeply below about $20 \mathrm{~dB} \mathrm{~S} / \mathrm{M}$. The figures also show that normalized error increases as the dynamic range of the spectrum decreases.

\section{CONCLUSTONS}

Although speech is intelligible even at $10 \mathrm{~W} S / N$, parameters extracted from speech vary significantly with $S / N$. We have shown that for vowel segments $S / N$ below 20 dB reduces dynamic range of spectrum and increases error in estimating parameters. For low level speech segments such as unvoiced sounds the $S / N$ will be very low making parametric extraction by I.P analysis extremely difficult.

\section{ACKNOWLEDGMENTS}

The authors wish to thank Prof. B.S. Remakrishne, Dr. V.V.S. Sarma and Nr. T.V. Ananthapadmanabha for many useful discussions.

\author{
REF BRENCES
}

1. D.R. Reddy, 'Speech Recognition by Machine:A Review', Proc. IEAE, Vol.64, No.6, p.501, Apri1 1976.

2. J. Pakhoul, 'Iinear Prediction: A Tutorial Review", Proc. IEE⿰, Vol.63, No. 4, p. 561, April 1975.

3. B. Yegnanarayana, "Fomant Extraction from Iinear Prediction Phase Spectra., (submitted). 